\title{
Repeatability of the three dimensional kinematics of the pelvis, spine and lower limbs while performing selected exercises
}

\author{
Maria Skublewska-Paszkowska ${ }^{1,}{ }^{*}$ Edyta Lukasik $^{1}$, Jakub Smołka ${ }^{1}$, Magdalena Zawadka ${ }^{2}$, Mirosław Jabłoński ${ }^{3}$, \\ and Piotr Gawda ${ }^{4}$ \\ ${ }^{1}$ Lublin University of Technology, Faculty of Electrical Engineering and Computer Science, Institute of Computer Science, Nadbystrzycka 38D, \\ 20-618 Lublin, Poland \\ ${ }^{2}$ Medical University of Lublin, Faculty of Health Sciences, Jaczewskiego 8 Street, 20-090 Lublin, Poland \\ ${ }^{3}$ Medical University of Lublin, Department of Rehabilitation and Orthopedics, Jaczewskiego 8, 20-090 Lublin, Poland \\ ${ }^{4}$ Medical University of Lublin, Faculty of Health Sciences, Department of Rehabilitation and Physiotherapy, Magnoliowa 2, 20-143 Lublin, \\ Poland
}

\begin{abstract}
Three-dimensional optical systems are used for calculating many kinetic and kinematic parameters. The obtained data are precise; however, their repeatability is a very important aspect. The aim of this paper is to verify the range of motion repeatability of one healthy subject in the joints of the pelvis, spine and lower limbs based on the coefficient of variation. The participant performed seven exercises, repeated five times: two-leg squat, single-leg squat, forward bending, forward-step motion, step onto the stair, hip extension in a standing position and tip-toe extension while standing. Motion was recorded using Vicon motion capture system consisting of eight NIR cameras. The participant had 39 markers attached to her body according to the Plug-in Gait model. The coefficient of variation was calculated in three dimensions (X, Y and Z). The greatest repeatability, pursuant to the coefficient, was observed during the two-leg squat and forward bending in the sagittal plane (X coordinate). It was also high during the singleleg squat. The lowest repeatability was observed during the tip-toe extension while standing and the hip extension in a standing position. During the step onto the stair and the forward-step motion, a higher repeatability of measurement occurred in the open kinematic chain than in the closed chain; in the hip extension the reverse occurred. Repeatability of a range of motion is different in two types of kinematic chain and in 7 exercises. Exercises such as tip-toe extension and hip extension, which require a greater ability to balance, indicated more variability in movement.
\end{abstract}

\section{Introduction}

Three-dimensional analyses are commonly used in biomechanical and clinical movement research to understand pathological movement patterns. Kinematic movement analysis has contributed valuable information to the physiology of human movement and physical therapy examination. It is also used to describe specific parameters of motor functionality.

Three-dimensional (3D) computerized motion analysis systems are routinely used in gait analysis research and in clinical practice, and have been applied in evaluating lower limb and lumbopelvic motion during functional tasks [1] .The use of various 3D systems for evaluating joints' kinematics has been previously investigated [2]. The VICON system is an optical tracking system which tracks three-dimensional movement trajectories of reflective markers with up to $1 \mathrm{~mm}$ accuracy. It is seen as the gold standard in optical gait analysis, and it is used for making comparisons with other systems [3-6].
Dynamic accuracy cannot be predicted based on static parameters. Previous studies revealed the significant influence of the system environment on the performance of video-based motion capturing systems. The number of cameras and the measurement site have the most significant influence on instrumental errors. The use of standard laboratory equipment makes the procedure widely applicable in functional error assessment [7-8]. Larger marker diameters led to a higher precision of measurement. Accuracy decreases significantly when using an optical lens filter. Optical motion capture provides precise measurement tools for biomechanical applications [9]. Marker placement, which differs from examiner to examiner, is identified as the largest source of variability in the evaluation of kinematic parameters.

Previous results demonstrate that system accuracy and reliability can be obtained allowing the collection of comparable data across different laboratories with varying configurations and equipment [10]. Vicon optical motion capture is accepted as a sensitive data research technology used for making comparisons with

Corresponding author: maria.paszkowska@pollub.pl 
new 3D motion systems [11]. The kinematic parameters of human gait measured using the Vicon system are some of most often parameters described in literature $[12,13]$. Motion capture systems were used for investigating running parameters [14,15]. However, there is a lack of kinematic analysis during exercises in open-closed chains.

The intra-class correlation coefficient of different methods of measuring the range of motion (goniometer, Vicon system and marker-less system) shows that the reliability of all methods is adequate. An alternative to Plug-in-Gait's model for calculating the center of a joint has also been proposed [16]. Electromagnetic motion capture systems also achieve a high level of repeatability in the analysis of pelvis and thorax kinematics in different environmental settings during a golf swing [17]. Because of motion capture usage in sport [18] and physical therapy research, the repeatability and variability evaluation of kinematic parameters are still needed to improve the quality of the physical measurement of human movement.

The purpose of this study is to use seven tests to evaluate the measurement repeatability of threedimensional motion analysis systems (using the Vicon optical motion capture system with the Plugin-gait model as an example). Seven different functional movement tasks (two-leg squat, single-leg squat, forward bending, forward-step motion, step onto the stair, hip extension in a standing position and tip-toe extension while standing) were recorded with $3 \mathrm{D}$ full-body kinematics.

To define the repeatability of joint kinematics measurement, range of motion (ROM) was selected. It was calculated as the difference between the maximum value of the angle and the minimum value of the angle. The authors take into account the ankle joint, the knee joint, the hip joint, the pelvic tilt and the spine.

\section{Materials and methods}

One young healthy woman (age 22, body length $1.76 \mathrm{~m}$, body mass $61 \mathrm{~kg}$ ) participated in this study. She made five repetitions of each of the following exercises:

- single leg squat with erect posture and legs held parallel,

- forward bending with extended knees,

- stepping onto a $16 \mathrm{~cm}$ high stair with one leg and stepping onto the stair (the participant started with toes $15 \mathrm{~cm}$ away from the step),

- full two-leg squat with upper limps held parallel to the ground (The participant squatted as low as she could safely, and then rose back up. The heels could not lift off the floor),

- forward-step motion, $30 \mathrm{~cm}$ in length and return-step motion,

- hip extension in standing position without trunk bending forward,

- maximal tip-toe ascent while holding her position for 3 seconds.

Approval for this research was obtained from both the ethical committee at the Lublin University of
Technology (no. 6/2015) and the ethical committee at the Medical University of Lublin (no. KE-0254/331/2015). The participant of the study was informed about the study procedures in detail. She signed a consent form prior to the measurements.

3D kinematic data was captured using a Vicon (Vicon Motion Systems Ltd., Oxford, United Kingdom) optical motion capture system equipped with eight NIR T40S cameras (two cameras mounted on each wall of the motion recording room) and two reference Bonita cameras on tripods. The Vicon Nexus 2.0 was used for data collection and post-processing. The data was sampled at $100 \mathrm{~Hz}$.

During a session the participant had 39 markers placed all over her entire body as specified in the Plug-in Gait Model documentation [19]. The markers were fixed to the skin using Vicon double sided tape. The participant was measured in compliance with the Plugin Gait Model specification (leg length, knee width, ankle width, shoulder offset, elbow width, wrist width, hand width, weight and height) so that subject created in Vicon Nexus software could be calibrated. All motion recordings were made in a room without daylight access. Ankle-joint angles were calculated using the Euler angle conventions of plantar flexion/ dorsiflexion about the $\mathrm{y}$-axis and varus/ valgus about the x-axis. The Plug-in Gait Model computed the subject kinematics and kinetics data. The measurements were input into the Vicon system so that the a new subject could be created. The first participant's capture was in a motorbike pose and was used for the subject's model calibration. Additionally, the marker placements were validated.

Recorded 3D data was post-processed: (1) each marker was labelled, (2) gaps in marker trajectories were filled, (3) the Plug-in Gait dynamic model was run. This computed the model outputs, some of which were used in this analysis. Then the recorded and generated data was exported to a C3D file (a file that contains the markers' positions and the model outputs for each frame of the recording). For this purpose a custom piece of software was written in $\mathrm{C}++$ using $\mathrm{B}-\mathrm{TK}$ (Biomechanical Toolkit) and Eigen libraries.

The software (figure 1) is used for automatic data export and collection. It (1) sets up variables containing input data details, values being exported, trial names etc., (2) goes through data recordings of consecutive objects and extracts their names (from file paths), (3) enumerates for each object all sessions (recording days) and extracts the session names, (4) enumerates trials and their variants, constructs the input file names and clears the temporary file list, (5) enumerates the exported values if the input file exists and saves them to temporary files, adds the names of those files to the temporary file list, (6) merges files in the temporary file list (to produce a single file with all the values for a particular trial), removes the temporary files. 


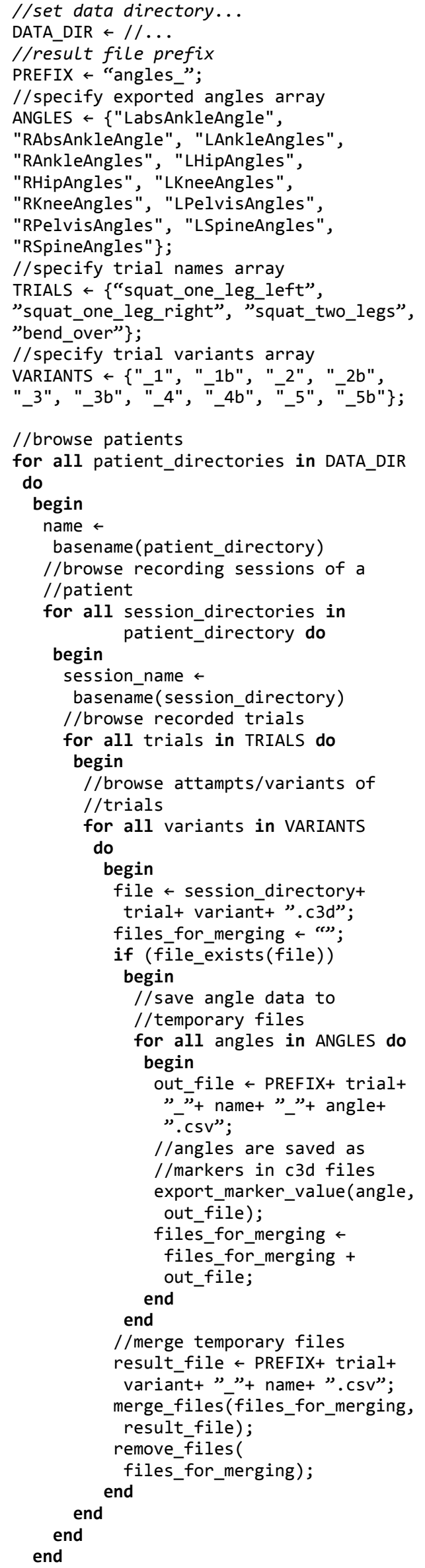

The coefficient of variation - CV (see formula 1) [20] was used for computing the repeatability of ROM.

$$
C V=\frac{\sigma}{\mu} \cdot 100
$$

where $\sigma$ denotes the standard deviation and $\mu$ the mean. An examined parameter was assumed to be repeatable when its $\mathrm{CV}$ was lower than ten.

\section{Results}

The greatest repeatability was observed during the twoleg squat and the forward bending in a sagittal plane (X coordinate). It was also high during the single-leg squat. The lowest repeatability was observed during the tip-toe standing and the extension of the hip.

Coefficient of variation $(\mathrm{CV} \%)$ was the highest in the transverse plane ( $\mathrm{Z}$ coordinate) in most exercises except for the tip-toe standing and single-right-leg squat, where the frontal plane motion had the worst repeatability.

Repeatability of a range of motion is different in the two types of kinematic chains and in 7 exercises. Exercises such as tip-toe extension and hip extension, which require a greater ability to maintain balance, indicated more variability in movement.

Specifically high repeatability, pursuant to the coefficient, was observed during the two-leg squat (Table 5) and the forward bending (Table 6) in a sagittal plane (X coordinate). The lowest repeatability was observed during the tip-toe extension while standing (Table 6) and the hip extension in a standing position (Table 4).

A higher repeatability of measurement occurred in open rather than in closed kinematic chains during stepping onto the stair and stepping forward. The same does not hold for hip extension in a standing position (the CV\% values are similar - Table 4).

However the range of motion was found to be highly repeatable in the saggital plane $(\mathrm{X})$ for all joints in the closed kinematic chain during the single-leg squat (the average of $\mathrm{CV} \%$ values for all joints is equal to $5,02 \%$ see Table 1). The average CV\% values for stepping forward and stepping onto the stair in the open kinematic chaing were slightly higher, equaling 6,64\% (Table 3) and $7,08 \%$ (Table 2 ) respectively.

\section{Discussion}

Repeatability of human gait parameters measured by the Vicon motion capture system was previously investigated using the coefficient of variation and the coefficient of multiple correlation [13, 14]. Intra-subject repeatability was excellent for kinematic data in the sagittal plane both within a test day as well as between test days. In our study joint movements in the sagittal plane were more repeatable than in the frontal or transverse planes motions.

Fig. 1. Pseudo-code used for data export and merging. 
Table 1. Coefficient of variation (\%) of range of motion during the single-leg squat (for each joint the upper row contains the values for the left side, the lower row contains values for the right side).

\begin{tabular}{|l|c|c|c|c|c|c|}
\hline \multirow{3}{*}{ Joints } & \multicolumn{6}{|c|}{ Single-leg squat } \\
\cline { 2 - 7 } & \multicolumn{2}{|c|}{ Open kinematic chain } & \multicolumn{2}{c|}{ Closed kinematic chain } \\
\cline { 2 - 7 } & $\mathrm{x}$ & $\mathrm{y}$ & $\mathrm{z}$ & $\mathrm{x}$ & $\mathrm{y}$ & $\mathrm{z}$ \\
\hline \multirow{3}{*}{ Knees } & 6.78 & 4.93 & 13.94 & 6.74 & 3.6 & 14.49 \\
\cline { 2 - 7 } & 3.15 & 0.85 & 6.08 & 1.92 & 21.55 & 4.57 \\
\hline \multirow{2}{*}{ Ankles } & 10.8 & 26.92 & 26.4 & 6.04 & 11.68 & 11.57 \\
\cline { 2 - 7 } & 36.02 & 12.4 & 10.55 & 3.79 & 18.86 & 18.32 \\
\hline \multirow{3}{*}{ Hips } & 4.37 & 7.72 & 22.02 & 5.69 & 7.21 & 11.01 \\
\cline { 2 - 7 } & 10.95 & 5.99 & 6.15 & 5.95 & 7.33 & 14.7 \\
\hline \multirow{2}{*}{ Mean } & 12.01 & 9.8 & 14.19 & 5.02 & 11.7 & 12.44 \\
\hline SD & 12.19 & 9.19 & 8.41 & 1.81 & 7.12 & 4.66 \\
\hline
\end{tabular}

Table 2. Coefficient of variation (\%) of range of motion during stepping onto the stair (for each joint the upper row contains the values for the left side, the lower row contains values for the right side).

\begin{tabular}{|l|c|c|c|c|c|c|}
\hline \multirow{3}{*}{ Joints } & \multicolumn{6}{|c|}{ Step onto the stair } \\
\cline { 2 - 7 } & \multicolumn{2}{|c|}{ Open kinematic chain } & \multicolumn{2}{c|}{ Closed kinematic chain } \\
\cline { 2 - 7 } & $\mathrm{x}$ & $\mathrm{y}$ & $\mathrm{z}$ & $\mathrm{x}$ & $\mathrm{y}$ & $\mathrm{z}$ \\
\hline \multirow{3}{*}{ Knees } & 5.34 & 2.45 & 11.12 & 28.68 & 16.59 & 11.27 \\
\cline { 2 - 7 } & 8.59 & 5.3 & 5.19 & 26.34 & 0.2 & 11.76 \\
\hline \multirow{3}{*}{ Ankles } & 11.3 & 13.58 & 13.24 & 16.73 & 21.24 & 21.12 \\
\cline { 2 - 7 } & 11.85 & 11.54 & 11.42 & 19.48 & 31.57 & 30.65 \\
\hline \multirow{3}{*}{ Hips } & 2.14 & 11.88 & 17.45 & 6.56 & 10.66 & 9.94 \\
\cline { 2 - 7 } & 3.29 & 20.65 & 12.21 & 6.64 & 43.25 & 43.2 \\
\hline \multirow{2}{*}{ Mean } & 7.08 & 10.9 & 11.77 & 17.4 & 24.94 & 21.32 \\
\hline SD & 4.11 & 6.42 & 3.96 & 9.44 & 11.57 & 13.32 \\
\hline
\end{tabular}

Table 3. Coefficient of variation (\%) of range of motion during forward step (for each joint the upper row contains the values for the left side, the lower row contains values for the right side).

\begin{tabular}{|l|c|c|c|c|c|c|}
\hline \multirow{3}{*}{ Joints } & \multicolumn{6}{|c|}{ Forward step } \\
\cline { 2 - 7 } & \multicolumn{2}{|c|}{ Open kinematic chain } & \multicolumn{2}{c|}{ Closed kinematic chain } \\
\cline { 2 - 7 } & $\mathrm{x}$ & $\mathrm{y}$ & $\mathrm{z}$ & $\mathrm{x}$ & $\mathrm{y}$ & $\mathrm{z}$ \\
\hline \multirow{3}{*}{ Knees } & 10.63 & 7.87 & 18.16 & 27.76 & 42.32 & 12.95 \\
\cline { 2 - 7 } & 5.35 & 4.98 & 6.04 & 8.45 & 18.9 & 12.17 \\
\hline \multirow{3}{*}{ Ankles } & 3.32 & 9.02 & 8.7 & 4.33 & 19.45 & 18.76 \\
\cline { 2 - 7 } & 8.57 & 11.47 & 11.15 & 3.08 & 13.86 & 13.73 \\
\hline \multirow{3}{*}{ Hips } & 7.69 & 13.17 & 35.64 & 8.87 & 18.9 & 26.48 \\
\cline { 2 - 7 } & 4.28 & 11.83 & 9.12 & 9.2 & 10.75 & 20.92 \\
\hline \multirow{2}{*}{ Mean } & 6.64 & 9.73 & 14.8 & 10.28 & 20.7 & 17.5 \\
\hline SD & 2.79 & 3.03 & 11 & 8.94 & 11.15 & 5.61 \\
\hline
\end{tabular}

Table 4. Coefficient of variation (\%) of range of motion during hip extension in a standing position (for each joint the upper row contains the values for the left side, the lower row contains values for the right side).

\begin{tabular}{|l|c|c|c|c|c|c|}
\hline \multirow{3}{*}{ Joints } & \multicolumn{6}{|c|}{ Hip extension } \\
\cline { 2 - 7 } & \multicolumn{2}{|c|}{ Open kinematic chain } & \multicolumn{2}{c|}{ Closed kinematic chain } \\
\cline { 2 - 7 } & $\mathrm{x}$ & $\mathrm{y}$ & $\mathrm{z}$ & $\mathrm{x}$ & $\mathrm{y}$ & $\mathrm{z}$ \\
\hline \multirow{3}{*}{ Knees } & 21.12 & 25.02 & 23.59 & 23.74 & 45.4 & 20.61 \\
\cline { 2 - 7 } & 41.03 & 70.39 & 61.57 & 17.65 & 16.05 & 18.82 \\
\hline \multirow{2}{*}{ Ankles } & 9.4 & 36.61 & 36.69 & 43.18 & 26.58 & 26.3 \\
\cline { 2 - 7 } & 31.83 & 33.36 & 33.36 & 46.41 & 22.82 & 21.83 \\
\hline \multirow{3}{*}{ Hips } & 6.52 & 33.93 & 53.21 & 11.16 & 22.97 & 28.8 \\
\cline { 2 - 7 } & 7.67 & 33.55 & 59.71 & 8.16 & 6.09 & 25.34 \\
\hline \multirow{2}{*}{ Mean } & 19.6 & 38.81 & 44.69 & 25.05 & 23.32 & 23.62 \\
\hline \multirow{2}{*}{ SD } & 14.34 & 15.96 & 15.62 & 16.24 & 13.03 & 3.8 \\
\hline
\end{tabular}

Table 5.Coefficient of variation (\%) of range of motion during two-leg squat (for each joint the upper row contains the values for the left side, the lower row contains values for the right side).

\begin{tabular}{|l|c|c|c|}
\hline \multirow{2}{*}{ Joint } & \multicolumn{3}{|c|}{ Two-leg squat } \\
\hline \multirow{2}{*}{ Knees } & $\mathrm{x}$ & $\mathrm{y}$ & $\mathrm{z}$ \\
\hline \multirow{2}{*}{ Ankles } & 2.95 & 3.05 & 7.45 \\
\cline { 2 - 4 } & 3.45 & 3.94 & 2.98 \\
\hline \multirow{2}{*}{ Hips } & 3.68 & 10.19 & 9.94 \\
\cline { 2 - 4 } & 4.15 & 17.98 & 18.43 \\
\hline Pelvis & 2 & 11.78 & 14.42 \\
\hline Spine & 2.06 & 17.47 & 14.72 \\
\hline Mean & 8.47 & 13.44 & 13.06 \\
\hline SD & 1.12 & 13.54 & 16.64 \\
\hline
\end{tabular}

Table 6.Coefficient of variation (\%) of range of motion during tip-toe standing and forward bending (for each joint the upper row contains the values for the left side, the lower row contains values for the right side).

\begin{tabular}{|l|c|c|c|c|c|c|}
\hline \multirow{2}{*}{ Joint } & \multicolumn{3}{|c|}{ Tip-toe } & \multicolumn{3}{c|}{ Forward bending } \\
\cline { 2 - 7 } & $\mathrm{x}$ & $\mathrm{y}$ & $\mathrm{z}$ & $\mathrm{x}$ & $\mathrm{y}$ & $\mathrm{z}$ \\
\hline \multirow{2}{*}{ Knees } & 15.56 & 20.85 & 8 & 10.03 & 8.01 & 10.88 \\
\cline { 2 - 7 } & 30.11 & 25.04 & 18.08 & 6.88 & 8.79 & 12.13 \\
\hline \multirow{2}{*}{ Ankles } & 1.76 & 6.6 & 6.37 & 6.81 & 25.54 & 24.26 \\
\cline { 2 - 7 } & 2.75 & 11.3 & 11.47 & 7.96 & 9.64 & 9.58 \\
\hline \multirow{2}{*}{ Hips } & 6.84 & 5.8 & 7.78 & 2.77 & 11.47 & 14.2 \\
\cline { 2 - 7 } & 22.99 & 96.91 & 38.21 & 3.22 & 11.45 & 7.32 \\
\hline Pelvis & 22.94 & 29.83 & 25.16 & 3.2 & 8.67 & 5.65 \\
\hline Spine & 16.23 & 37.09 & 77.75 & 1.73 & 8.15 & 9.13 \\
\hline Mean & 14.9 & 29.18 & 24.1 & 5.33 & 11.46 & 11.64 \\
\hline SD & 10.34 & 29.55 & 24.24 & 2.98 & 5.85 & 5.75 \\
\hline
\end{tabular}


The marker-based MoCap system has high reliability and validity as indicated by the coefficient of variation (CV\%) [21]. Another study provides evidence that a marker-based and a markerless protocol report similar ranges of pelvis and lower limb angles in the single-leg squat [22]. The knee's range of motion was under special consideration because of the frequency of injuries in sport activities. The repeatability of knee motion measurement turned out to be the lowest during squat and lung exercises when compared to eleven motor tasks [23]. In our study we analyzed the hip, the ankle and the knee joints and the pelvic and the spine motion, and we observed the highest repeatability during the two-leg squat and the forward bending in a sagittal plane. Because the hip extension and the tip-toe extension require balancing and holding one's position, there is more variation in the range of motion than in the case of others exercises. More stable exercises, especially with two-leg support have the highest repeatability of movement.

The joint angle can be adjusted without incurring any changes in other joints in an open kinematic chain. There is one primary joint axis and a single dominant plane of movement during the exercise. Moreover, more isolated joint motion occurs. In a closed kinematic chain both ends of the chain are fixed and any changes in the angle of one joint reciprocally results in altered angles in the other joints. Triplanar multiple-joint movement occurs. Complex movements such, as stepping onto the stair, lunging, stepping forward, typically consist of both open and closed kinematic chains. When one lower limb is fixed to the ground, the other moves freely.

Previously, the parameters of the lower limbs and pelvis were correlated between kinematic chains during gait [24]. Our study shows that open kinematic chains have higher repeatability of movement, probably because the main part of the motion occurred in this chain. Legs in the closed kinematic chain have to maintain balance and give stable support. Because the range of motion in closed kinematic chains was small in most cases, the relative change values may seem large.

\section{Conclusions}

Repeatability of the joint range of motion may be affected by the type of exercise, the stability of the position and the type of kinematic chain. Further investigations of tridimensional motion are needed to explain the differences observed between closed and open kinematic chains.

\section{References}

1. A. G. Schache, P.D. Blanch, D,A. Rath, T.V. Wrigley, R. Starr, K. L. Bennell. Hum. Mov. Sci. 21, 273-293 (2002)

2. M. Kim, C. Yi, O. Kwon, S. Cho, H. Cynn, Y. Kim, S. Hwang, B. Choi, J. Hong, D. Jung. Spine 38, 1260-1267 (2013)
3. Y. Levanon, A. Gefen, Y. Lerman, U. Givon, N. Z. Ratzon. Gait Posture 32, 469-474 (2010)

4. B. Müller, W. Ilg, M. A. Giese, N. Ludolph. PLOS ONE 12, e0175813 (2017)

5. S. Springer, G. Y. Seligmann. Sensors 16, 194 (2016)

6. L. D. Duffell, N. Hope, A. H. McGregor. Proc. Inst. Mech. Eng. H (2014)

7. P. Eichelberger, M. Ferraro, U. Minder, T. Denton, A. Blasimann, F. Krause, H. Baur. J. Biomech. 49, 2085-2088(2016)

8. G. E. Gorton III, D. A. Hebert, M. E. Gannotti. Gait Posture 29, 398-402 (2009).

9. M. Windolf, N. Götzen, M. Morlock. J. Biomech. 41, 2776-2780 (2008)

10. E. Miller, K. Kaufman, T. Kingsbury, E. Wolf, J. Wilken, M. Wyatt. Gait Posture 50, 116-119 (2016)

11. K. Otte, B. Kayser, S. Mansow-Model, J. Verrel, Friedemann Paul, A. U. Brandt, T. Schmitz-Hübsch. PLoS ONE 11, (2016)

12. M. P. Kadaba, H. K. Ramakrishnan, M. E. Wootten. J. Orthop. Res. 8, 383-392 (1990)

13. M. P Kadaba, H. K. Ramakrishnan, M. E. Wootten, J. Gainey, G. Gorton, G. V. B. Cochran. J. Orthop. Res. 7, 849-860 (1989)

14. A. G. Schache, P.D. Blanch, D,A. Rath, T. V. Wrigley, R. Starr, K. L. Bennell. Gait Posture 15, 136-145 (2002)

15. D. Levine, M. A. Colston, M. W. Whittle, E. C. Pharo, D. J. Marcellin-Little. J. Athl. Train. 42, 29 34 (2007)

16. S. P. Nair, S. Gibbs, G. Arnold, R. Abboud, W. Wang. Clin. Biomech. Bristol Avon 25, 582-587 (2010)

17. VICON, 2016. Plug-in Gait Product Guide [WWW Document]. VICON. URL http://www.vicon.com/downloads/documentation/vi con-documentation/plug-in-gait-product-guide (accessed 26.06.2017)

18. J. Wasik, GB Shan. ARCHIVES OF BUDO 11, 6167, (2015)

19. JF. Reed, F. Lynn. Meade, BD. Clin Diagn Lab Immunol 9 (6) 1235-1239 (2002)

20. K. Evans, SA. Horan, RJ. Neal, RS. Barrett, PM. Mills. Sports Biomech. 11, 262-272 (2012)

21. M. Yazdifar, M. Yazdifar. Eur. Sci. J. ESJ 11, (2015) http://eujournal.org/index.php/esj/article/view/5979

22. M. A. Perrott, T. Pizzari, J. Cook, J. A. McClelland. Gait Posture 52, 57-61 (2017)

23. K. Desloovere, P. Wong, L. Swings, B. Callewaert, H. Vandenneucker, A. Leardini. Gait Posture 32, 597-602 (2010)

24. Z. Svoboda, M. Janura, P. Kutilek, E. Janurova. J. Hum. Kinet. 51, 37-43 (2016) 